\title{
Improving the Value Proposition of Surveillance Tools: Innovative Uses for VA ESSENCE
}

\author{
Cynthia A. Lucero-Obusan ${ }^{\star 1}$, Patricia Schirmer ${ }^{1}$, Gina Oda ${ }^{1}$ and Mark Holodniy ${ }^{1,2}$
}

'Department of Veterans Affairs, Washington, DC, USA; ${ }^{2}$ Stanford University, Stanford, CA, USA

\section{Objective}

To describe VA's experience developing innovative and alternative uses of a surveillance system and improve the overall value proposition of this tool for the agency.

\section{Introduction}

VA began using ESSENCE as a public health surveillance tool in 2005. The system offered alerting capability for pre-defined syndromes and querying capability for outpatient ICD-9 diagnosis codes. Herein, we highlight examples of how we have invested in upgrades to analytic capabilities and expanded data sources available to ESSENCE in order to augment the overall utility of this system within VA.

\section{Methods}

We reviewed use cases for new data added since 2009 (additional outpatient, plus inpatient, procedure, surgery, and telephone triage data) as well as enhancements to system analytics, geospatial mapping and general functionality. We also describe novel use cases for the original outpatient data elements and document examples of how we've merged query results from ESSENCE with data from other VA data sources to answer important surveillance questions.

\section{Results}

The evaluation was divided into three themes. First, and a top priority, was enhancing influenza surveillance capability. A number of innovative use cases for this theme were reviewed. Pulling procedure codes (ICD-9 and CPT) provided the ability to track immunizations in outpatient and inpatient settings. The availability of outpatient clinic location details allowed us to calculate \%ILI specifically for our primary care clinics. Telephone triage data gave us more timely insight into the rise and peak of influenza activity compared to outpatient visit data alone. The addition of inpatient data assisted us in characterizing the severity of each season by tracking admitting and discharge diagnosis codes for influenza as well as other severity elements, such as ward details (intensive care stays), overall lengths of stay, mechanical ventilation requirements (via intersecting time series of influenza diagnosis query and mechanical ventilation procedure query) and patient disposition (specifically, deaths) for influenza-coded hospitalizations. New geospatial capabilities enabled us to map hospitalizations and telephone triage calls by VA facility and region. Finally, we merged our inpatient ESSENCE data with pharmacy and laboratory data from other VA data sources to assess the proportion of hospitalized Veterans who had influenza testing performed and/or received antiviral medications.

The second theme was epidemiologic reviews and lookbacks. Here we sought to identify Veterans with an exposure or procedure of interest. Most recently, we conducted a review of Veterans with Carbapenem-resistant Enterobacteriaceae (CRE) infections having undergone specific endoscopy procedures (Endoscopic Retrograde Cholangiopancreatography, ERCP). We queried for ERCP procedure codes in ESSENCE then merged this with CRE laboratory data to generate a cohort of patients requiring further epidemiologic review.

The third theme was infection control activities. Here, in partnership with facility Infection Preventionists, we identified procedures, events or diagnoses of interest for specific surveillance questions. We used procedure codes in ESSENCE to identify operative procedures defined by the National Healthcare Safety Network to generate Surgical Site Infection (SSI) surveillance denominators. In another example, we sought to assess whether patients with healthcare-associated pneumonia were being appropriately tested for Legionella. We used inpatient diagnosis codes to identify pneumonia hospitalizations in ESSENCE and merged that with laboratory data as a starting point for the analysis. Finally, at the request of our local accident review committee, we used existing outpatient diagnosis codes in ESSENCE to identify needlestick injury visits that may not have been properly reported.

\section{Conclusions}

We've highlighted new and innovative uses of a public health surveillance tool within VA. System evaluations such as these are essential for demonstrating usefulness as well as maintaining funding and support for these applications. Through the mechanisms described, we have optimized the functionality of this tool to the greatest extent possible. In order to continue to innovate and harness the power of big data available to us, we have begun transitioning to a new surveillance platform (Praedico, Bitscopic Inc.) which provides additional flexibility and system architecture needed to accommodate the volume of data available from multiple sources and improve our analytic, event detection, geospatial and forecasting capabilities into the future.

\section{Keywords}

Public Health Surveillance; Veterans; System Enhancement; Influenza surveillance; Epidemiology

\section{*Cynthia A. Lucero-Obusan \\ E-mail: cynthia.Iucero@va.gov}

